\title{
Recent Developments in Ultra-High Speed and Large Area Photomultiplier Tubes
}

\author{
James Milnes ${ }^{1}$, Tom Conneely and Jon Howorth \\ Photek Ltd \\ 26 Castleham Road, St Leonards-on-Sea, East Sussex, TN38 ONR UK \\ E-mail: james.milnes@photek.co.uk
}

\section{Colin Horsfield, Michael Rubery, Stefan Parker and Dianne Hussey $A W E$}

Aldermaston, Reading, Berkshire, RG7 4PR UK

Fusion diagnostics can involve the measurement of ultra-fast optical pulses, either generated by Cherenkov radiation $(<1 \mathrm{~ns})$ or fast scintillator crystals $(\sim \mathrm{ns})$. At NIF the dynamic range of the signal to be measured can cover up to 19 orders of magnitude with a duration of about 2 ns.

Photek has been working with AWE to provide photomultiplier (PMT) solutions for fusion diagnostics. By using microchannel plates (MCP) as the gain medium, the time response of the PMTs can be as fast as 100 ps FWHM. We will present data showing developments in the accurate measurement of the true impulse response of the fastest detectors. We will also show improvements in the pulse uniformity as a function of illuminated area and the solution to a peculiar saturation effect.

Fusion diagnostics that utilise high speed scintillators often need to capture a large area of light with a high degree of time accuracy. Microchannel plate (MCP) photomultiplier tubes (PMT) are recognised as the leading device for capturing fast optical signals. However, when manufactured in their traditional proximity focused construction, the time response performance is reduced as the active area increases. This is due to two main factors: the capacitance of a large anode and the difficulty of obtaining small pore MCPs with a large area. Collaboration between Photek and AWE has produced prototype devices that combine the excellent time response of small area MCP-PMTs with a large active area by replacing the traditional proximity-gap front section with an electro-optically focused photocathode to MCP. We will present the first results from a new $100 \mathrm{~mm}$ diameter device with a pulse FWHM of $\sim 350 \mathrm{ps}$.

First EPS Conference on Plasma Diagnostics - $1^{\text {st }}$ ECPD

14-17 April 2015,

Villa Mondragone, Frascati (Rome) Italy

\footnotetext{
${ }^{1}$ Speaker
} 


\section{Introduction}

Fusion diagnostics can involve the measurement of ultra-fast optical pulses, either generated by Cherenkov radiation $(<1 \mathrm{~ns})[1]$ or fast scintillator crystals $(\sim \mathrm{ns})$ [2]. At the National Ignition Facility [3] (NIF) the dynamic range of the signal to be measured can cover up to 19 orders of magnitude with a duration of about $2 \mathrm{~ns}$. Often signals of interest are in close temporal proximity to much larger events, and we have recently published work demonstrating the improved gating ability of our devices [4].

Photek has been working with AWE to provide a photomultiplier (PMT) solution for fusion diagnostics. By using microchannel plates (MCP) as the gain medium, the time response of the PMTs can be as fast as 100 ps FWHM. The range of detectors available covers PMT models with three, two, one or no MCPs which give gain of $3 \times 10^{7}, 10^{6}, 5 \times 10^{3}$ or unity respectively. A combination of detectors each covering a dynamic range of $\sim 50 \mathrm{~V}$ into a $50 \Omega$ load (for an impulse response) right down to the noise floor of the measurement oscilloscope provides a very large measurement range.

\section{Pulse Shape}

\subsection{Accurate Pulse Shape Measurements}

A key performance parameter of PMTs used in fusion research is the speed of the pulse output, as accurate temporal tracking of the processes during the reaction is critical. We have previously inferred a pulse response of a single MCP, $10 \mathrm{~mm}$ active diameter PMT (PMT110) to be less than 90 ps FWHM [5] but recent advances in high bandwidth, real-time oscilloscopes have made direct measurements possible.

Photek quote the FWHM of the pulse output of a standard PMT110 as being $110 \mathrm{ps}( \pm 10 \mathrm{ps})$, but our measurement system is limited by the width of our optical source (Photek LPG -45 ps FWHM) and the bandwidth of the sampling oscilloscope (Agilent 86100C \& 54754A module $18 \mathrm{GHz}$ ). Both of these will broaden the response of the measured output, particularly the laser pulse width.

AWE were able to offer access to a short pulse laser at the Orion Laser Facility of $\sim 4$ ps at a rate of $10 \mathrm{~Hz}$, as well as a $45 \mathrm{GHz}$ LeCroy Wavemaster $845 \mathrm{Zi}$-A oscilloscope. As this device is real time, it removed the requirement for a very low jitter reference signal needed when using a sampling oscilloscope. However, as the output connector of the small Photek detectors is SMA as standard, the output signal is still limited to $18 \mathrm{GHz}$. We also measured the pulse output of the Photek photodiode PD010, essentially the same device but without the MCP providing any electron gain. The difference in the observed result is clearly seen in Figure 1. As expected, the more dramatic difference is observed in the faster PD010, and as the devices are otherwise identical this also gives an indication of the temporal spreading influence of the MCP. The comparison also indicates an after-pulse in the Photek LPG amongst the ringing in the trailing edge, as the AWE laser source is believed to be relatively clean in this regard.

\subsection{Pulse Shape as a Function of Area}

Another development that Photek have recently introduced is to modify the anode to improve the consistency of the pulse shape as a function of the illuminated area of the PMT. 
Previous observations have shown that the PMT would still respond outside of the defined $10 \mathrm{~mm}$ active area diameter. This would not necessarily be a problem, however it was effecting the nature of the ripple on the trailing edge, leading to a variation in the pulse shape depending on which part of the PMT was illuminated. To demonstrate this effect, the 45 ps Photek laser pulse stimulation was focused to a $\sim 1 \mathrm{~mm}$ diameter spot and scanned across the PMT input window, and the result is shown in Figure 2 (a).
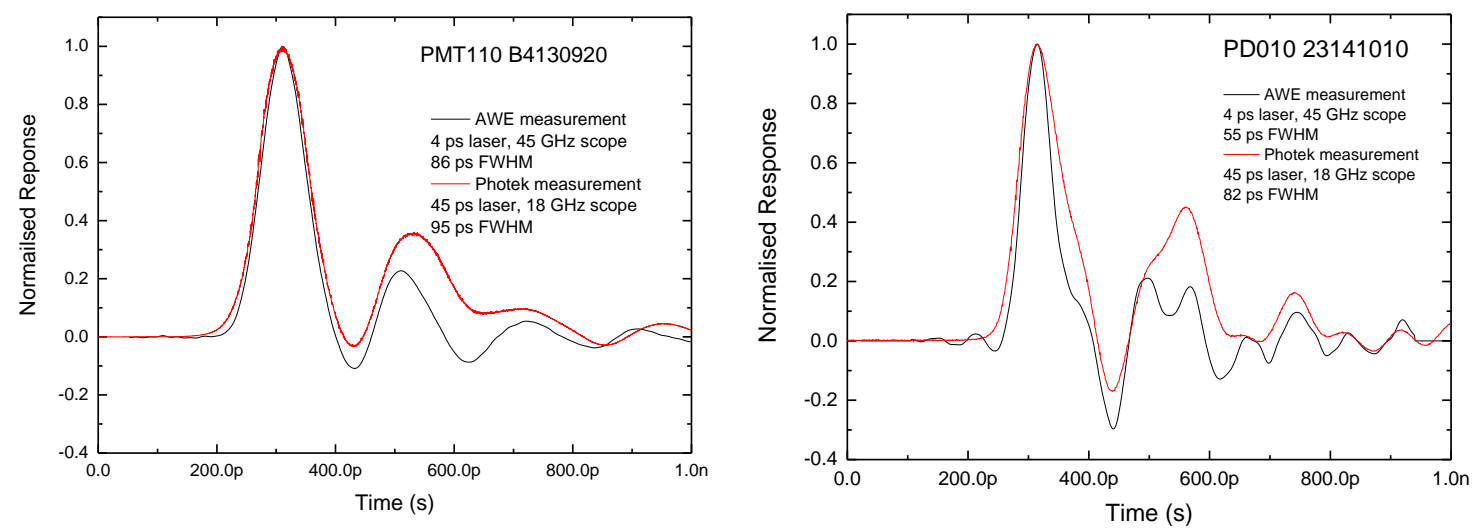

Figure 1: Pulse output comparisons for a PMT (left) and photodiode (right) showing the influence of the laser pulse width and measurment bandwidth on the observed result.

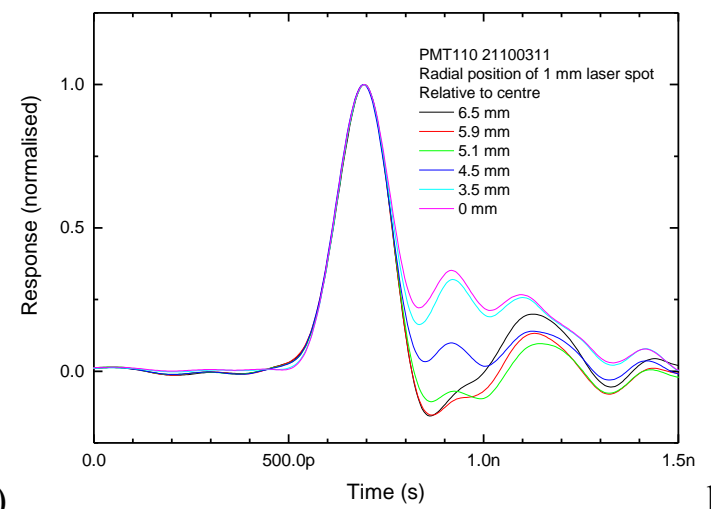

a)

Figure 2: The influence on the trailing edge ripple as a function of the location of the optical illumination without an anode mask (a) and with an anode mask (b)

The PMT was still responding to light outside of the active area because the two active internal components - photocathode and $\mathrm{MCP}$ - are both larger than $10 \mathrm{~mm}$ diameter. Therefore, to negate this effect, we introduced a mask around the anode that matched the $10 \mathrm{~mm}$ diameter active area. The results are shown on Figure 2 (b). On the device with a reducing aperture, the amplitude of the pulse response beyond a $5 \mathrm{~mm}$ radius fell away very quickly. The oscillations in the trailing edge will be a combination of the after-pulse from the Photek LPG previously mentioned and that inherent in the detector, which can vary marginally from device to device. The aim of this modification was to introduce consistency over the active area. All traces were averaged, stable and repeatable. 


\subsection{Pulse Shape as a Function of Gain}

As mentioned previously, in fusion diagnostics PMTs often have to deal with a large variation in signal levels. The ability to adjust the electron gain over several orders of magnitude is a very useful aspect of PMTs, however we needed to verify that the pulse output was consistent throughout the working range of gain available. To acheive this a PMT was illimunated by a floodfill laser pulse and the gain was changed without altering the input power of the laser. The result is shown on Figure 3.

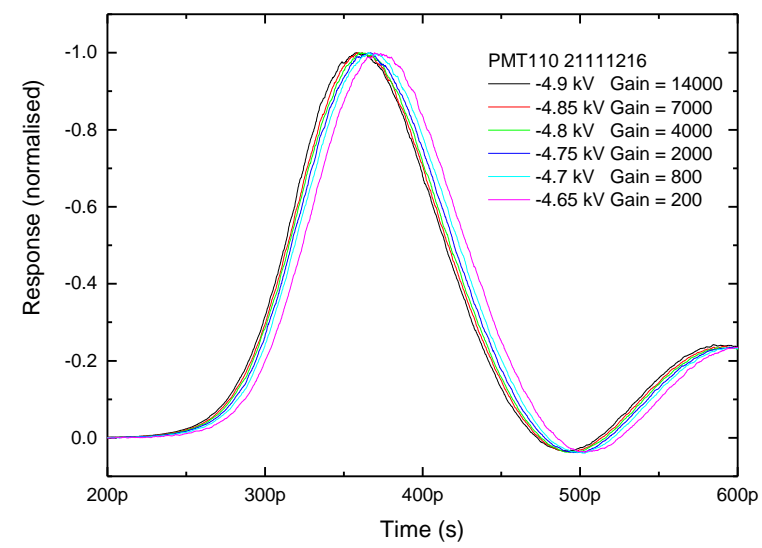

Figure 3. Pulse output of a PMT110 for a range of electron gain values.

Figure 3 shows that the pulse shape appears to be very consistent, but with a noticable delay in the pulse arrival time at low gain, which we can atribute to the nature of the electron avalanche within the MCP pores. A closer inspection of the 50\% transition times of the leading and trailing edges, and the corresponding effect on the FWHM, is shown on Figure 4.
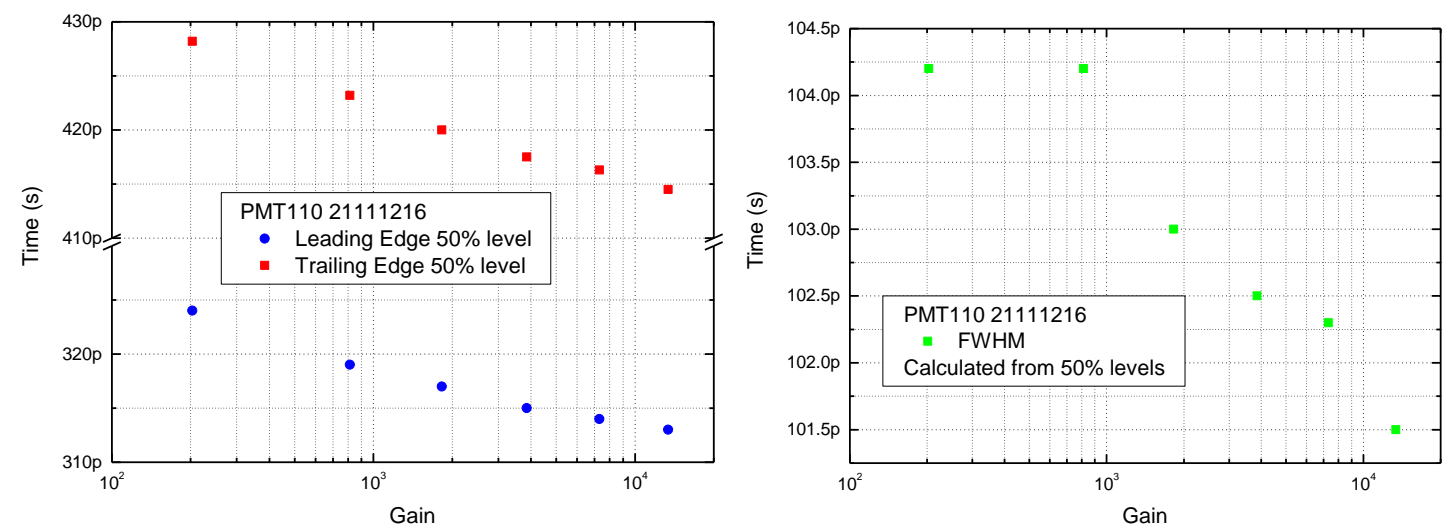

Figure 4. The influence of PMT gain on the leading and trailing edge 50\% transition times (left) and the corresponding influence on the FWHM (right).

While the arrival time of the output pulse varies by $\sim 10$ ps over 2 orders of magnitude of operating gain, the pulse FWHM only varies by $\sim 3$ ps.

\section{Pulse Saturation}

In addition to monitoring the performance of the PMTs over a range of output levels, we have also examined how the output changes with a variable input magnitude. This work has been looking at pulse widths in the range of a few nanoseconds (ns), rather than the near-impulse 
responses examined so far, because any on-set of non-linearity is much easier to spot in this regime.

The main issue that was observed in early models was an unexpected increase in signal level during a pulse width of several ns, and the effect was worse as the signal level was increased. This is demonstrated on Figure 5 where a $40 \mathrm{~mm}$ diameter PMT with one MCP (PMT140) is illuminated with a roughly square pulse input with a width of $60 \mathrm{~ns}$, and the power input is increased by reducing the ND filters between the laser source and the PMT. It is clear that the output shape is changing as the input level is increasing, and this became known as the "ramp" effect.

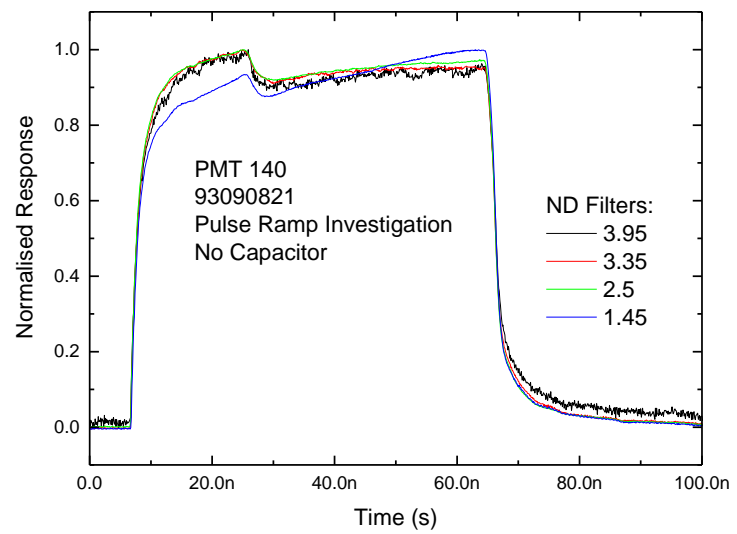

Figure 5: A demonstation of the "ramp" effect in an older model PMT140.

It was speculated that this could be due to the large amount of charge being extracted from the MCP causing a voltage transient on the MCP output face, temporarily increasing the gain of the MCP. To negate this effect a high voltage capacitor was added between MCP output and $0 \mathrm{~V}$, and the results are shown on Figure 6 (a).
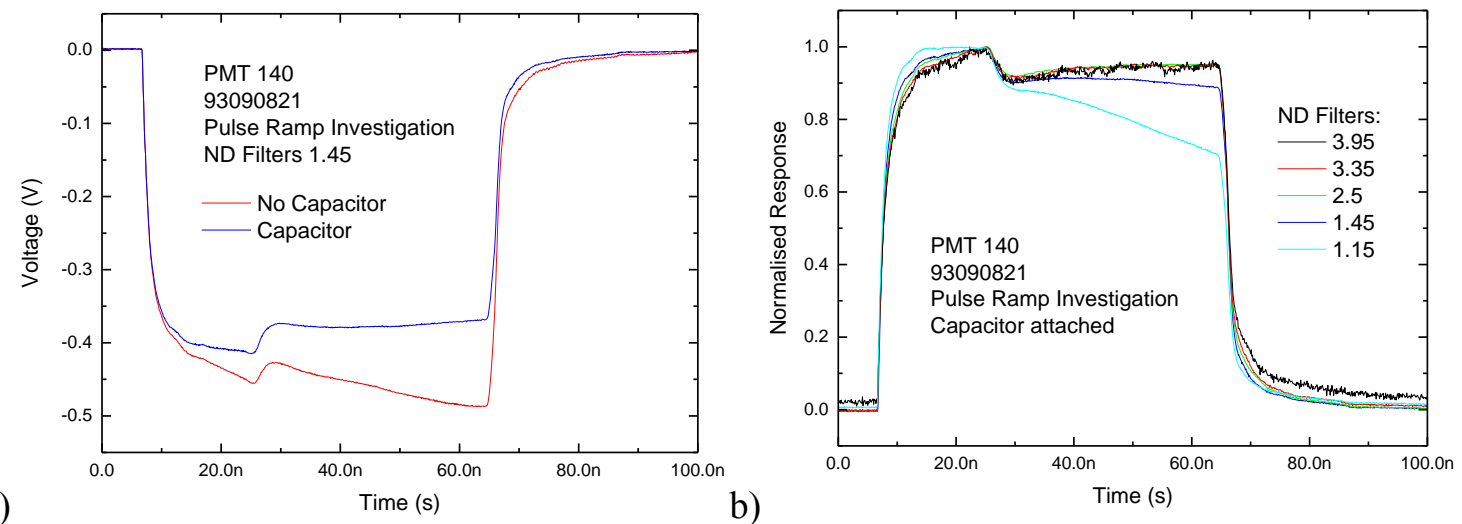

Figure 6: The removal of the "ramp" effect through the addition of a capacitor to the MCP output.

Once the capacitor was added, the PMT was shown to saturate in a more intuitive manner as shown in Figure 6 (b) with the PMT output reducing towards the end of the pulse as the charge store is exhausted. The capacitor is now standard in all Photek PMTs. The next stage will be to do a full analysis showing the onset of saturation as a function of input power, area of illumination and pulse width. 


\section{Large Area PMT Development}

Depending on the signal available, distance from the source and the focusing geometry, sometimes PMTs need to have a large active area. However, the pulse shape of standard large area ( 25 or $40 \mathrm{~mm}$ diameter) MCP-PMTs is noticeably inferior to their small area $(10 \mathrm{~mm})$ equivalents. The large anode capacitance gives a slow and often oscillating trailing edge, and small pore $(3 \mu \mathrm{m})$ MCPs that provide the best speed are only available with $18 \mathrm{~mm}$ working diameter.

A possible solution is to replace the proximity gap between the photocathode and MCP with an electro-static focusing section, as shown on Figure 7 (a). This would be able to combine the anode \& MCP assembly of a small PMT with the working area of a large PMT. Previous work has demonstrated this on a single and double MCP device with $40 \mathrm{~mm}$ active diameter [6], and we are currently analysing a double MCP, $100 \mathrm{~mm}$ active diameter prototype. An inital result showing a promising pulse width of just 350 ps FWHM is shown on Figure 7 (b).

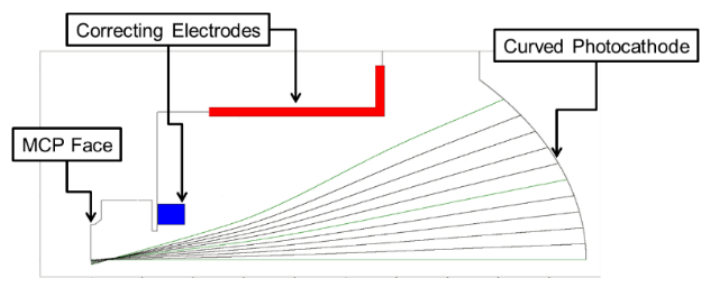

a)

Figure 7 (a) A focusing section replaces the traditional proximity gap between photocathode and MCP in a large area PMT. (b) Preliminary pulse shape data for a double MCP, $100 \mathrm{~mm}$ active diameter PMT.

\section{Summary}

We have improved the accuracy of the impulse response measurements of our fast detectors, with our $10 \mathrm{~mm}$ photodiode showing a true response of $55 \mathrm{ps} \mathrm{FWHM} \mathrm{and} \mathrm{the} \mathrm{corresponding} \mathrm{PMT}$ giving 86 ps FWHM. We have enhanced the pulse response uniformity over the working area of the detector, and corrected some saturation effects. Preliminary data on a novel $100 \mathrm{~mm}$ active diameter PMT shows a pulse FWHM of 350 ps.

\section{References}

[1] H. W. Herrmann et al., Cherenkov radiation conversion and collection considerations for a gamma bang time/reaction history diagnostic for the NIF, Rev. Sci. Instrum. 79, 10E531 (2008).

[2] V. Yu. Glebov et al., Prototypes of National Ignition Facility neutron time-of-flight detectors tested on OMEGA, Rev. Sci. Instrum. 75, 3559 (2004).

[3] E. I. Moses, The National Ignition Facility: Status and Plans for the Experimental Program, Fusion Sci. Technol, 44, 11 (2003).

[4] J. S. Milnes et al., Ultra-high speed photomultiplier tubes with nanosecond gating for fusion diagnostics, Rev. Sci. Instrum. 83, 10D301 (2012).

[5] C. J. Horsfield et al., Development and characterization of sub-100 ps photomultiplier tubes, Rev. Sci. Instrum. 81, 10D318 (2010).

[6] J. S. Milnes et al., Improved time response for large area microchannel plate photomultiplier tubes in fusion diagnostics, Rev. Sci. Instrum. 85, $11 \mathrm{E} 601$ (2014). 